\title{
An Analysis of Human Error in Skill Task and Visual Recognition under Auditory Interruption
}

\author{
Futoshi Sugimoto, Toru Toyabe and Akira Sato \\ Department of Information and Computer Sciences, Toyo University \\ 2100, Kujirai, Kawagoe, Saitama 350, Japan \\ Tel : $+81-492-39-1448$ \\ E-mail : f_sugi@(cc.eng.toyo.ac.jp
}

\begin{abstract}
When we drive a car. we have to do different kinds of works simultaneously. and thus wc are apt to make some errors under such simulaneous works. In recent years. there have been many drivers who use a cellular phone while driving a car. And there happen some car accidents that are caused by the use of the cellular phone. This kind of accidents has become one of the social problems today. To use of a hands-free phone is efficient to free the driver from the operation of a cellular phone or from looking aside while driving. which may cause an accident. However. there is a critical possibility for the driver to lose his attention and misjudge. even if he uses a handsfree phone. This might be said to be a kind of a hidden dangerous phenomenon. When a driver communicates with someone by a phone, he has to do three extra works that he does not need to do in a usual driving. One of them is to listen to and to understand the person whom he is talking to. and the second is to think of the answer to lim. and the third is to talk to him.

In our research experiment we have set an experimental environment consisting of three tasks on a computer screen to quantitatively analyze mutual effect of these tasks. The first task is a tracking task. which is a typical skill base one. The second task is to recognize the visual information that is indicated by four different media on a computer screen and to respond as soon as possible. The third task is to listen to a conversation and answer a question about the conversation. This task is supposed to be the same as using a cellular phone while driving a car.

In this paper we have examined the effect of third task on the first and the second tasks. There was not any effect of the third task on the performance of the tracking task. The time needed to respond after getting the information was affected a little by the third task. The number of overlooking the visual information in the second task significantly increased by the effect of the third task. This point might be one of the causes that result in a car accident.
\end{abstract}

\section{INTRODUCTION}

The operators working for a plant. the pilot of an airplane. and. on a more immediate level. the drivers of a car. have to do different kinds of works carefully and simultaneously. They are apt to make some errors under 0-7803-4053-1/97/\$10.00 1997 IEEE such simultaneous works. where it is rather difficult for them to concentrate their attention and they sometimes lack their attention itself. Matsui [1] and Kleiman [2] have modeled a way of how to optimally distribute human attention on an information field to get as much of the information as possible. There are some research papers published on the relation between human attention and human error. In these studies, the attention was defined as the finite resources and was supposed to have the same quality on the whole. We. however. had better think that the characteristics of attention and the form of its distribution differ according to what kind of work it is. So we suggested to classify a recognition of visual information into two groups; an active type of recognition and a passive one. and we proposed a model where a different kind of attention was distributed to each type of recognition with a different form [3].

In recent years. many people have become to use a cellular phone. and there are a number of drivers who use a cellular phone while driving a car. Therefore there happen some car accidents. whose causes may be thought of using a cellular phone while driving. This kind of accident has become one of the social problems today. In some countries. they never let a person drive while using a cellular phone. except a hands-free one. under the law: This may be one of the immediate solutions. But we do not think it is a complete answer to the problem. To use of a hands-free phone is efficient to free the driver from the operation of a cellular phone or from looking aside while driving. which may cause an accident. for example. However. there is a critical possibility for the driver to lose his attention and misjudge, even if he uses a handsfree phone. This might be said to be a kind of a hidden dangerous phenomenon.

A driver has to do three extra works. which he does not need to do in a usual driving. when he communicates with other person by the cellular phone. The first is to listen to and to understand the person whom he is talking to. and the second is to think of the answer to him. and the third is to talk to him. Sometimes the driver does these with a person seated next to him. But in such a case. the person seated next to the driver also understands the traffic, and he does not enforce a more critical conversation upon the driver. which otherwise may cause an accident. On the other hand, a person whom the driver is talking to on the phone does not know the traffic. thus involuntarily enforces to do those three duties upon the driver. 
To consider an efficient measure to decrease the accidents. it is very important to quantitatively grasp what kind of and how much of effect the works of thinking and talking have on driving a car and judging the traffic. In this paper we have modeled how to drive a car. how to recognize the traffic. and how to talk on the phone while driving a car as simplified tasks.

We have set an experimental environment consisting of three tasks on a computer screen to analyze mutual effect of these works quantitatively. The first task is a tracking task. which is a typical skill base task. The second task is to recognize the visual information that is indicated by four different media on a computer screen. and to respond as soon as possible. The media are consisted of an inorganic pattern. an emotional pattern. color and a movement. The inorganic pattern is of the geometric one that does not have any semantics. The emotional pattern is of the cartoon face that has facial cxpressions such as anger or pleasure. Each medium indicates four separate informations that are encoded in four ways. The third task is to listen to a conversation and to answer a question about the contents of the comversation. This task is supposed to be the same as using a cellular phone while driving a car.

In this paper we have examined the effect that the third task has on the first and the second tasks. There was not any effect of the third task on the performance of the first task. The time needed to respond in the second task lad a little effect. and it took a little longer when the third task was added. The number of overlooking the visual information in the second task was significantly increased by the effect of the third lask. This point might be one of the causes that result in a car accident.

\section{AN EXPERIMENTAL TASK FOR A MODEL OF DRIVING}

When we apply some simultaneous works of driving to Rasmussen's model of information processing [4]. there appear three levels of works as below. Driving a car is a skill base work because it is a pattern of action highly automated. and it is also unconsciously controlled under intention to smoothly move a car. According to a pattern of distributing attention to correctly recognize the traffic. we can divide it into two types of recognition: an active type of recognition and a passive one. In the passive way: the recognition is driven by a stimulus and the attention is distributed unconsciously to some indefinite field. so we may regard it as the work near a skill base one. but not completely. On the other hand. the active way is a rule base work. because we control our attention consciously in such a work that is actively going to get the specified information. Finally. those three works. that is. listening. considering and talking that are done in a conversation by a cellular phone. are knowledge base tasks. In this way. as the works in three levels of information processing. we have modeled the simultaneous works of driving and the other ones that are interrupted and added by using a cellular phone. In our experiment we have respectively assigned a simple task to every abstract work in the model as shown in Table 1. For example. we make a subject do the tracking task that

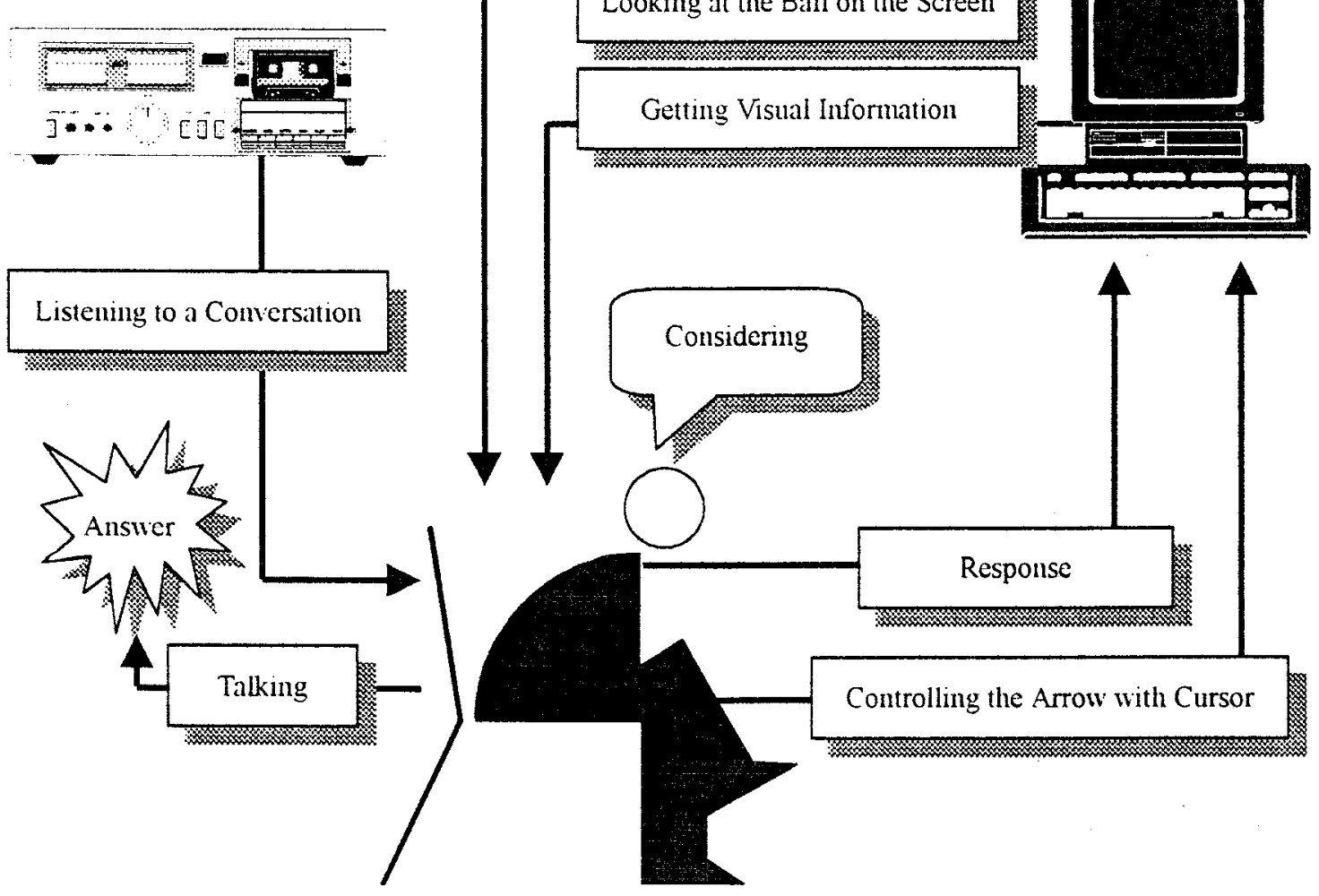

Fig. 1 Diagram of Experiment 
Driving a Car

Recognizing the Traffic

Talking on the Cellular Phone
Skill Work

Visual Recognition

Auditon Information Processing
Tracking Task

to Find and Respond

to Listen and Answer

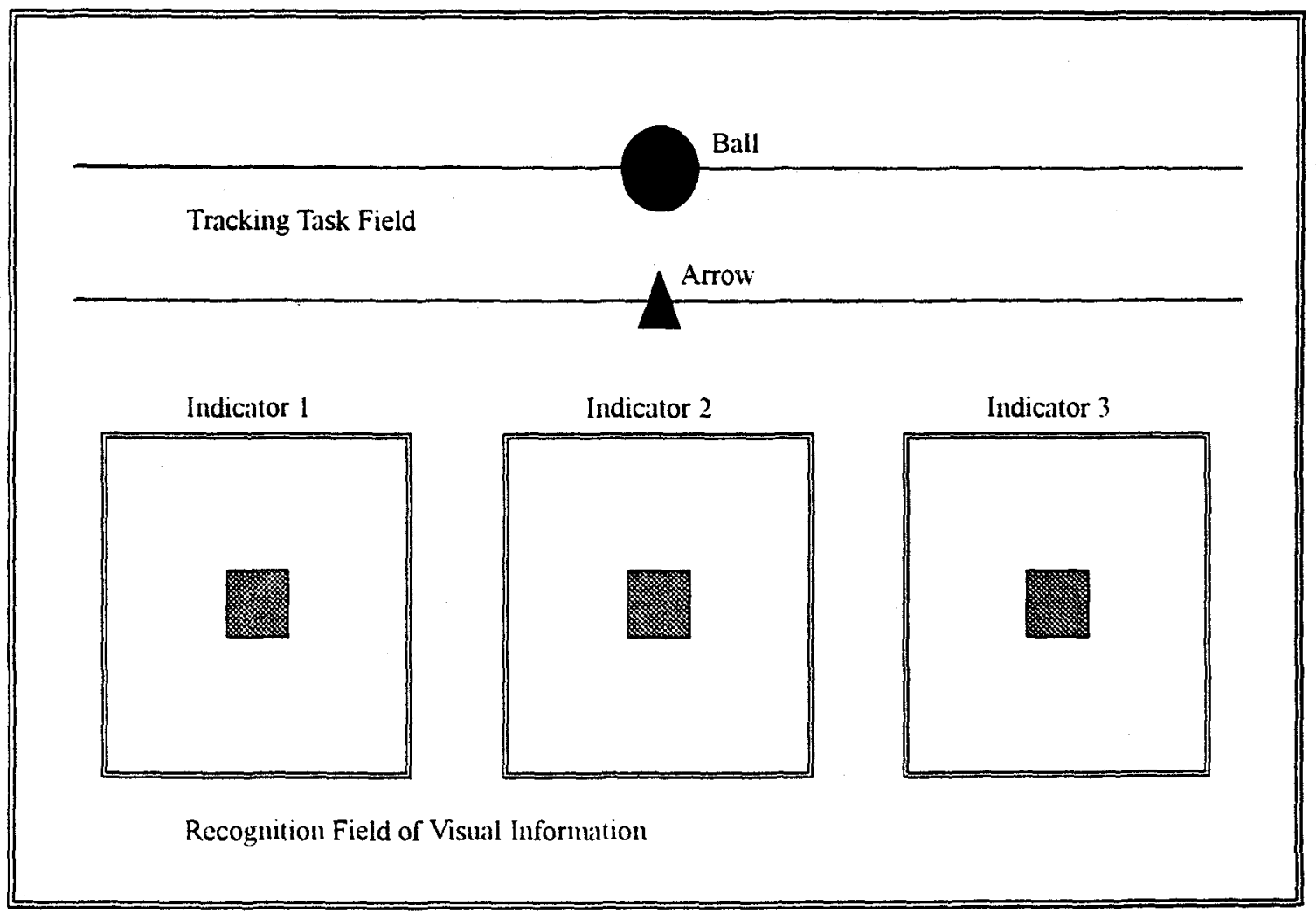

Fig. 2 Computer Screell of Experiment

is a typical skill base task for a driving operation. Furthenmore. we make a subject recognize information. instead of the traffic. on a computer screen at random. We are showing our conception diagram of the experiment in Figure 1

\section{EXPERIMENTAL ENVIRONMENT}

\section{Tracking Task (the First Task)}

The upper part of Figure 2 shows a working field of the tracking task. A ball in the upper section moves from side to side. and the ball is controlled by saw waves shown in Figure 3. A subject has to make the arrow of the lower section follow the novement of the ball by operating the right cursor ker and the left one with his right hand. There are three kinds of movement of the ball as the saw waves of Figure 3 show. (a) This is a simple coming and going from the right edge to the left. (b) The turning point changes at random. but the speed is the same as the first one. (c) The speed of the movement is faster than the second one. The tracking task becomes relatively more difficult from (a) to (b). and from (b) to (c). The subject has to carry out three kinds of the tracking task respectively: The performance of the tracking task is evaluated by the accuracy of making the arrow follow the ball.

\section{Visual Recognition (the Second Task)}

The lower pan of Figure 2 shows a working field to recognize the visual information. where there are three indicators. A scries of information is indicated in a random position. at random seconds of interals and in 


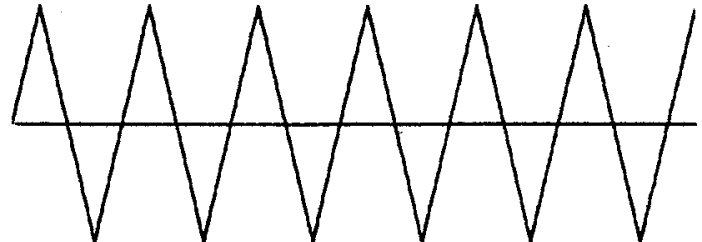

(a)

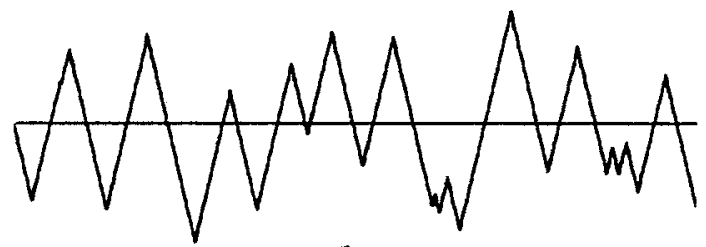

(b)

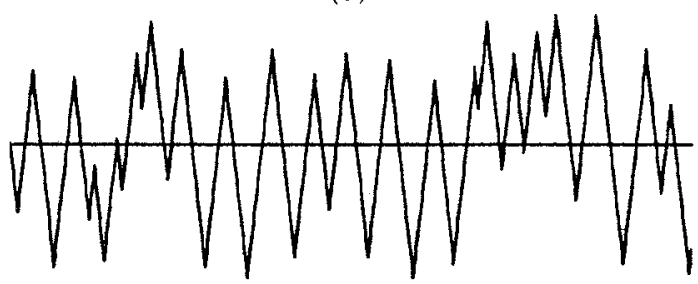

(c)

Fig. 3 Saw Wave Controlling the Ball

random sequence for just one second. by four kinds of media that are encoded in four ways as shown in Table 2. The media are consisted of an inorganic pattern. an emotional pattern. color and a movement. The inorganic patten is of the geometric one that does not have any semantics. The emotional pattern is of the cartoon face that has facial expressions such as anger or pleasure. Each medium indicates four separate informations that are encoded in four ways. As the information of a code in a medium is indicated four times on an indicator. the total of the indication comes to 192 times +4 codes $x+$ media $x 3$ indicators $x+$ times). The interal indicating the information is set at random between three and six seconds. The four media have been prepared to know whether there is any difference of the performance to recognize the information. but we do not argue the point in this paper.

The subject is expected to respond by pushing a key with his left hand as soon as he recognizes any information shown on any indicators. The performance of this task is evaluated by the time needed to respond. which is measured by 10 milli-second unit. If the subject does not respond within two seconds after the presentation of the information. we suppose he overlooks the information.

\section{Auditory Information Processing (the Third Task)}

This task acts as internuption to the first and the second task. We make the subject listen to a Japanese conversation for approximately twenty seconds through headphones and then the subject is asked a question about the conversation. The subject has fifteen seconds to answer the question. $\mathrm{He}$ is requested to answer it in a sentence. not in words. The series of listening to the conversation and answering the question is contimued as the subject doing the first and the second task. The experiment was carried out in two cases. without the third task and with the third task respectively: And we examined the effect of the third task over the first and the second tasks.

\section{RESULT OF EXPERIMENT}

\section{Error Rate of the Tracking Task}

When the arrow is more apart from the ball than a constant distance. which is two times of diameter of the ball. we consider it to be the error. An error rate of the tracking task is calculated every interval by equation (1). and the average of them is the error rate of the subject.

$$
\text { error rate }=\frac{\text { time of error }}{\text { time of interval }}
$$

The average of nine subjects' error rates is plotted in Figure 4 . (a) shows a difference between the error rate according to the difficulty of the tracking task. (b) shows a difference between the error rate in the case that there is the third task or not. The error rate became higher in proportion to the difficulty of the tracking task. This result is necessary. and it is not surprising at all. But it is to be noted that there is no effect of the third task in each tracking task of different difficulty. The knowledge base task does not affect to the skill base task at all.

Table 2 Information Media and Their Codes

\begin{tabular}{lllll}
\hline Information Media & & Information Code & \\
\hline Inorganic Pattern & Circle & Triangle & X mark & Wave \\
Emotional Pattern & Pleasure & Anger & Surprise & Sorrow \\
Color & Green & Red & Blue & Yellow \\
Moveinent & Up & Right & Down & Left \\
\hline
\end{tabular}




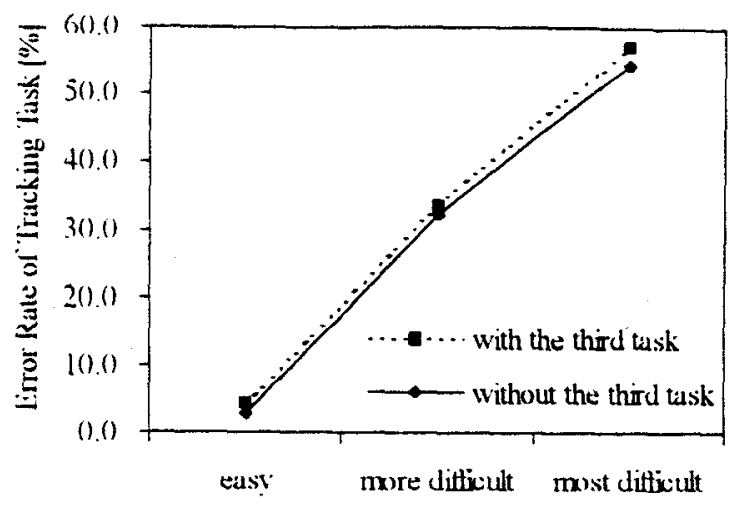

(a)

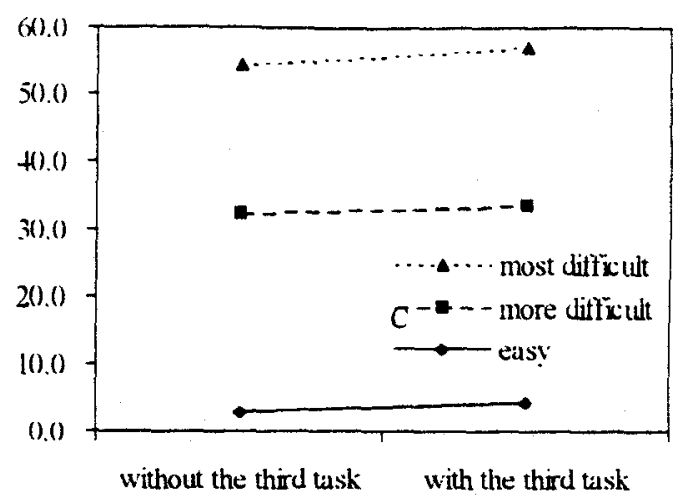

(b)

Fig.t Error Rate of Tracking Task

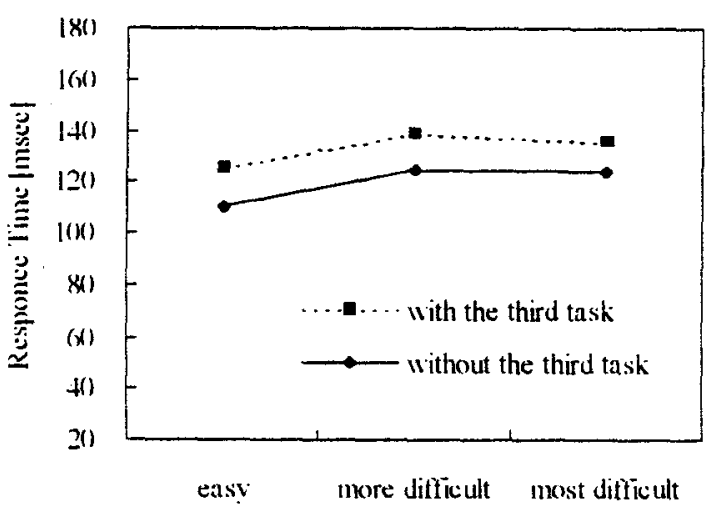

(a)

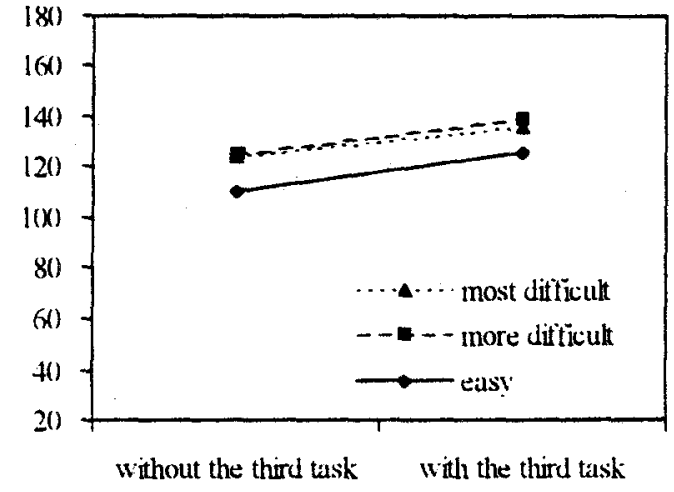

(b)

Fig. 5 Response Time in Visual Recognition

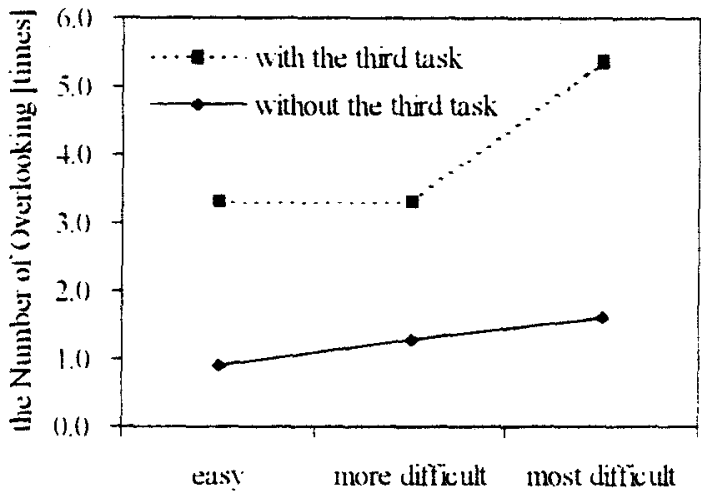

(a)

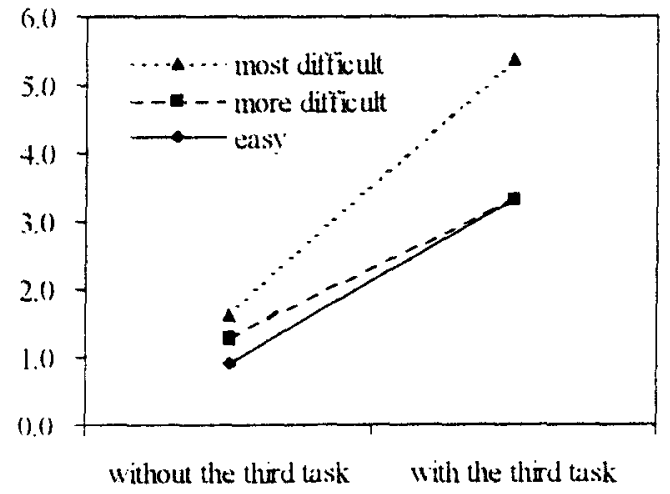

(b)

Fig.6 the Number of Overlooking in Visual Recognition

\section{Time Needed to Respond}

The time needed for the subject to respond is measured by 10 milli-second unit. The responded time is measured only when the subject responds in 192 times of information prescutation. The average of ever responded time is a performance of the subject. The average time of nine subjects is plotted in Figure 5 in the same form as Figure + . The time needed to respond is not affected by the variation of the difficulty in the tracking task. But it is affected a little by the third task. The time 
needed to respond takes a little longer when the third task is added

\section{Overlooking Visual Information}

In the second task. if the subject does not respond within two seconds after the information presentation. we suppose it is overlooking. The average number of orerlooking by nine subjects is plotted in Figure 6 in the same form as Figure + and Figure 5 . No effect is appeared with a variation of the difficulty in the tracking task in the same way as the time needed to respond. But the effect of the third task was noticed most. When the second task is divided into the work to perceive the information and into the work to respond by pushing a key: it is supposed that the former is nearer to the rule base work. the latter is nearer the skill base work. The skill base task is hard to receive the effect of the knowledge base task. and the nule base task is easy to receive.

\section{CONCLUSION}

We have showed the result of our experiment and lave described only the facts resulted from the experiment in the previous section. In this section. basing on the experimental result. we try to consider the relation between the attention and the human crror in simultaneous works. We don't need to pay much attention in the skill base work. The perfonmance of the work greatly depends on a physical skill. especially in the case of the tracking task. As the difficulty in the skill base work increased. its own performance fell down in proportion to its difficulty: but no effect appeared on the other bases. On the contrary. additioning of the knowledge base work also had no effect over the performance of skill base work. This fact shows that the knowledge base work does not share the attention resources with the skill base work at all.

As mentioned above. the recognition of the risual information in this experiment was of passive type. and it could be divided into two sub-works. One of them. responding. is nearer to the tracking task. so it did not conflict with the knowledge base work so much as the tracking task did. But the work to perceive information conflicted with the knowledge base work very much. This experimental result means that if only the subject notices the indication of information. he would ordinarily respond. and he would lose his attention to notice for the knowledge base work.

Considering an actual driving. it may be guessed that the overlooking of information. which rarely happens. causes a car accident. We have not investigated the active type of recognition in this paper. It may be said that this type of recognition will be remarkably affected by the knowledge base work. The effect of using a cellular phone differs according to what level of recognition the driver needs. So. we need to investigate the level of recognition of a person who actually drives a car.

\section{REFERENCES}

[1] N. Matsui. "Evaluative Cognition and Attention Allocation in Human Interface." Trans. Erect. Info. Com. Eng. Vol. J70-D. No. 11. Nov. 1987. pp.23212326.

[2] D. Kleiman. "Solving the Optimal Attention Allocation Problem in Manual Control." Trans. on Automatic Control. Vol. AC-21. No. 6. Dec. 1976. pp.813-822

[3] F. Sugimoto. "Performance Evaluation of Display Media. Color. Pattern. and Movement in Passive Type of Cognitive Process." Proceedings of IECON'95. 1995. pp. 1062-1067.

[4] J. Rasmussen. "Skill. Rules. and Knowledge. Signals. Signs. and Symbols. and Other Distinctions in Human Perfornance Models." Trans. on Systems. Man. and Cybernetics. Vol. SMC-13. No. 3. Mar. 1983. pp.257266. 